\title{
BOUNDARY VALUE PROBLEMS FOR FUNCTIONAL DIFFERENTIAL EQUATIONS
}

\author{
BY ROBERT FENNELL ${ }^{1}$ AND PAUL WALTMAN ${ }^{2}$
}

Communicated by Wolfgang Wasow, October 21, 1968

Let $E^{n}$ denote Euclidean $n$ space with norm $|\cdot|$ and let $C_{h}$ denote the space of continuous $E^{n}$ valued functions on $[a-h, a], h>0$, with uniform norm $\|\cdot\|$. For a function $x(t)$ on $[a-h, b]$ and $t \in[a, b]$ let $x_{t}$ denote the function on $[a-h, a]$ whose value at $\theta$ is $x(t+\theta-a)$. Let $f(t, \Psi)$ be a mapping from $[a, b] \times C_{h}$ into $E^{n}$ and let $M$ and $N$ be linear operators from $C_{h}$ to $C_{h}$. In this paper we consider special cases of the boundary value problem

$$
\begin{aligned}
y^{\prime} & =f\left(t, y_{t}\right), \\
M y_{a}+N y_{b} & =0, \quad b>a+h .
\end{aligned}
$$

This is a nonlinear version of a problem posed by Cooke [1]. Other boundary value problems for functional differential equations have been studied recently by Grimm and Schmitt [3], Halanay [4], and Kato [6].

We treat the problem for bounded $f$ and a restricted class of operators by initial value methods, that is, we seek to find an initial function $q \in C_{h}$ such that a solution of the initial value problem (1) and

$$
y(t)=q(t), \quad a-h \leqq t \leqq a
$$

satisfies the boundary condition (2). Some functions $f\left(t, y_{t}\right)$, not bounded, can be treated by approximation techniques and some nonhomogeneous boundary conditions can also be considered.

THEOREM 1. Let $f(t, \Psi)$ be a continuous bounded function from $[a, b] \times C_{h}$ into $E^{n}$ and let $M$ and $N$ be $n \times n$ matrices such that $M+N$ is nonsingular. If $\left\|(M+N)^{-1} N\right\|<1$, then there exists a solution of (1) and (2).

Method of Proof. We give a brief sketch of the method of proof of Theorem 1. The proofs of the other theorems are similar. Assume first that solutions of the initial value problem are unique. Let $T: C_{h} \rightarrow C_{h}$ be defined as follows: for $q \in C_{h}$, let $T q=x_{b}(q)$, that is, $T q$ is the segment at $b$ of the solution of the initial value problem with

\footnotetext{
1 Research of this author supported by a NASA Traineeship.
}

2 Research of this author supported by Project Themis. 
initial data $q$. Let

$$
\begin{aligned}
S(\alpha, L)=\left\{q\left|q \in C_{h},\|q\| \leqq \alpha,\right|\right. & \left|q\left(\theta_{1}\right)-q\left(\theta_{2}\right)\right| \\
& \left.\leqq L\left|\theta_{1}-\theta_{2}\right|, \theta_{1}, \theta_{2} \in[a-h, a]\right\} .
\end{aligned}
$$

The operator

$$
\begin{aligned}
F(q) & =\left[I-(M+N)^{-1}(M+N T)\right] q \\
& =-(M+N)^{-1} N(T-I) q
\end{aligned}
$$

is shown to have a fixed point $q^{*} \in S(\alpha, L)$ for an appropriate choice of $\alpha$ and $L$. The solution of the initial value problem with initial data $q^{*}$, satisfies the boundary condition (2). An approximation argument, essentially that of Kato [6], is then used to remove the uniqueness assumption.

In case the matrix $M$ is invertible the boundary condition (2) can be written

$$
y_{a}+P y_{b}=0 \text {. }
$$

If $\|P\|<1$, then $I+P$ is nonsingular $[2, \mathrm{p} .62]$ but it is not necessarily the case that $\left\|(I+P)^{-1} P\right\|<1$. The following theorem covers this case.

THEOREM 2. Let $f(t, \Psi)$ be a continuous bounded function from $[a, b] \times C_{h}$ into $E^{n}$ and let $P$ be an $n \times n$ matrix. If $\|P\|<1$, then the boundary value problem (1) (4) has a solution.

In the preceding theorems, the desired initial function was selected from a compact set $S(\alpha, L)$. We can replace the matrix $P$ in Theorem 2 by a linear operator but we no longer can select the initial condition from a predetermined compact set and thereby lose the approximation argument which allowed the assumption of unique solutions of the initial value problem (1) (3) to be avoided.

Theorem 3. Let $f(t, \Psi)$ be a continuous bounded function from $[a, b] \times C_{h}$ into $E_{n}$ and let $P$ be a continuous linear operator from $C_{h}$ into $C_{h}$ with $\|P\|<1$. Suppose solutions of the initial value problem are unique. Then there exists a solution of (1) (4).

THEOREM 4. Let the hypotheses of Theorem 3 hold with the boundedness assumption on $f(t, \Psi)$ replaced by a Lipschitz condition. If $e^{L(b-a)}(b-a)<(1-\|P\|) / L\|P\|$, there exists a unique solution of (1) (4).

Details will appear elsewhere. 


\section{REFERENCES}

1. K. L. Cooke, Some recent work on functional-diferential equations, Proc. U. S.-Japan Seminar on Differential and Functional Equations (Minneapolis, Minn.), Benjamin, New York, 1967, pp. 27-47.

2. V. N. Faddeeva, Computational methods of linear algebra, Dover, New York, 1959.

3. L. S. Grimm and K. Schmitt, Boundary value problems for delay-differential equations, Bull. Amer. Math. Soc. 74 (1968), 997-1000.

4. A. Halanay, On a boundary value problem for linear systems with a time lag, J. Differential Equations 2 (1966), 47-56.

5. - Differential equations stability, oscillations, and time lags, Academic Press, New York, 1966.

6. J. Kato, Asymptotic behavior in functional differential equations, Tohoku Math. J. 18 (1966), 174-215.

University of Iowa, Iowa City, Iowa 52240 